\title{
Establishing the Response of Low Frequency Auditory Filters
}

Menachem Rafael of [National Institute of Aerospace], Andrew Christian, Kevin Shepherd, Stephen Rizzi

[NASA Langley Research Center], James Stephenson [US Army Aviation Development Directorate]

The response of auditory filters is central to frequency selectivity of sound by the human auditory system. This is true especially for realistic complex sounds that are often encountered in many applications such as modeling the audibility of sound, voice recognition, noise cancelation, and the development of advanced hearing aid devices. The purpose of this study was to establish the response of low frequency (below $100 \mathrm{~Hz}$ ) auditory filters. Two experiments were designed and executed; the first was to measure subject's hearing threshold for pure tones (at 25, 31.5, 40, 50, 63 and $80 \mathrm{~Hz}$ ), and the second was to measure the Psychophysical Tuning Curves (PTCs) at two signal frequencies ( $\mathrm{Fs}=40$ and $63 \mathrm{~Hz}$ ). Experiment 1 involved 36 subjects while experiment 2 used 20 subjects selected from experiment 1. Both experiments were based on a 3-down 1-up 3AFC adaptive staircase test procedure using either a variable level narrow-band noise masker or a tone. A summary of the results includes masked threshold data in form of PTCs, the response of auditory filters, their distribution, and comparison with similar recently published data. 